Instituto Internacional de Investigación y Desarrollo Tecnológico Educativo INDTEC, C.A.

DOI: https://doi.org/10.29394/Scientific.issn.2542-2987.2021.6.19.18.349-365

OAI-PMH: http://www.indteca.com/ojs/index.php/Revista Scientific/oai

Ensayo Original / Original Essay

\title{
Turismo Agroecológico: La integración entre lo presencialidad y la virtualidad
}

\author{
Autores: Iraima Sofía Gutiérrez Mendoza \\ Universidad Nacional Experimental de los Llanos Occidentales Ezequiel Zamora, UNELLEZ \\ gunenaeli@gmail.com \\ Barinas, Venezuela \\ https://orcid.org/0000-0003-0832-9663 \\ Yamirle Mercedes Pérez Girón \\ Universidad Nacional Experimental de los Llanos Occidentales Ezequiel Zamora, UNELLEZ \\ ympg1234@gmail.com \\ Barinas, Venezuela \\ https://orcid.org/0000-0003-4253-1718
}

Antonio José Gutiérrez Mendoza Instituto Nacional de Capacitación y Educación Socialista, INCES gutierrezm65@gmail.com

Cojedes, Venezuela https://orcid.org/0000-0001-6465-8360

\section{Resumen}

En un siglo XXI donde la educación universitaria atraviesa turbulencias, la Universidad Nacional Experimental de los Llanos Occidentales Ezequiel Zamora (UNELLEZ), Barinas, Venezuela, no queda excluida de los desafíos presentes. La actualidad tiene como arista la tecnología, modificando las formas de aprendizajes en los estudiantes siendo más visuales e interactivos. Es ahí, donde la revolución cognoscitiva por medio de la creación e innovación de formas llamativas entra en el terreno universitario para llamar la atención de los jóvenes hacia los contenidos de aprendizajes. La germinación de carreras nuevas hacia la virtualidad se hace necesaria, la presencia de un docente facilitador, el cual, mediante la interacción, transforme los conocimientos en preguntas para despertar el razonamiento en los estudiantes y ayudarlos a encontrar las respuestas a sus inquietudes. El objetivo del ensayo propone en ofrecer una reflexión sobre cambio significativo en los procesos enseñanza-aprendizaje virtual en el Programa de Formación de Grado Licenciatura en Turismo Agroecológico (PFGLTA), donde se aprende haciendo conllevando a un aprender aprendiendo, análisis, reflexión mediante las experiencias y las vivencias logrando familiarizarse con la adquisición de nociones virtuales mediante las herramientas digitales para palpar las realidades sensibles del entorno del Turismo Agroecológico, conquistar el interés en el campo turístico.

Palabras clave: educación universitaria; educación a distancia; aprendizaje en línea; enseñanza-aprendizaje; turismo agroecológico.

Cómo citar este ensayo:

Gutiérrez, I., Pérez, Y., \& Gutiérrez, A. (2021). Turismo Agroecológico: La integración entre lo presencialidad y la virtualidad. Revista Scientific, 6(19), 349-365, e-ISSN: 2542-2987. Recuperado de: https://doi.org/10.29394/Scientific.issn.2542-2987.2021.6.19.18.349-365

Fecha de Recepción: 21-08-2020
Fecha de Aceptación: 19-12-2020
Fecha de Publicación: 05-02-2021 
Instituto Internacional de Investigación y Desarrollo Tecnológico Educativo INDTEC, C.A.

DOI: https://doi.org/10.29394/Scientific.issn.2542-2987.2021.6.19.18.349-365

OAI-PMH: http://www.indteca.com/ojs/index.php/Revista Scientific/oai

Ensayo Original / Original Essay

Agroecological Tourism: The integration between face-to-face and virtuality

\begin{abstract}
In a 21st century where university education is going through turbulence, the Ezequiel Zamora National Experimental University of the Western Llanos (UNELLEZ), Barinas, Venezuela, is not excluded from the present challenges. The current edge is technology, modifying the ways of learning in students being more visual and interactive. It is there, where the cognitive revolution through the creation and innovation of striking forms enters the university field to draw the attention of young people to the contents of learning. The germination of new careers towards virtuality is necessary, the presence of a facilitator teacher, who, through interaction, transforms knowledge into questions to awaken reasoning in students and help them find the answers to their concerns. The objective of the essay proposes to offer a reflection on significant change in the virtual teaching-learning processes in the Bachelor's Degree Training Program in Agroecological Tourism (PFGLTA), where you learn by doing leading to learning by learning, analysis, reflection through experiences and the experiences becoming familiar with the acquisition of virtual notions through digital tools to touch the sensitive realities of the environment of Agroecological Tourism, gain interest in the tourist field.
\end{abstract}

Keywords: university education; long distance education; online learning; teaching-learning; agroecological tourism.

How to cite this essay:

Gutiérrez, I., Pérez, Y., \& Gutiérrez, A. (2021). Agroecological Tourism: The integration between face-to-face and virtuality. Revista Scientific, 6(19), 349-365, e-ISSN: 2542-2987. Recovered from: https://doi.org/10.29394/Scientific.issn.2542-2987.2021.6.19.18.349-365

Date Received: 21-08-2020
Date Acceptance:

19-12-2020
Date Publication: 05-02-2021 


\section{Ensayo Original / Original Essay}

\section{Introducción}

La realidad del siglo XXI al término del primer decenio, una sociedad cambiante a gran velocidad, conceptos en boca de todos como educación en línea/e-learning, videoconferencias/webinar, teletrabajo, clases/carreras virtuales, dado origen a la creación de entornos con características descentralizadas mediante la combinación de herramientas, aplicaciones de la Web.

Las universidades enfrentan nuevos retos, carreras llamativas para la continuación de estudios universitarios con jóvenes visuales, interactivos. Se hace notorio, la comprensión de fenómenos: tecnología, desarrollo sustentable, innovación por nombrar los más resaltantes en la actualidad, los cuales, debe estar presente en el enfoque de nuevos ofrecimientos académicos. A consideración, no existe un modelo exitoso y único de la enseñanza universitaria, es un abanico de variables contextuales para la decisión de la enseñanza.

En el plano virtual, existen gamas de posibilidades donde los docentes pueden cambiar significativamente la interacción enseñanza-aprendizaje, implicando nuevas formas de aprender, pensar, comunicar, trabajar debido a las tecnologías. Abriendo espacios flexibles en los cuales, los jóvenes sean partícipes dinámicos contextualizando escenarios de enseñanza-aprendizaje desde lo presencialidad y la virtualidad.

El surgimiento de nuevas opciones universitarias no puede perderse el horizonte de la conexión entre facilitadores, estudiantes, internet, contenidos programáticos, unidades curriculares y la metodología didáctica. Innegablemente, dentro del sistema educativo universitario actual, cada día, son más efectivos los esfuerzos hacia una educación digital integral entre recursos tecnológicos y naturales, elementos fundamentales en un siglo XXI, donde prevalece la información, conocimientos, tecnología, pero también, el bienestar ambiental. 
Dando énfasis a lo recalcando por la Organización de las Naciones Unidas para la Educación, la Ciencia y Cultura (UNESCO, 1998): en su informe mundial de la educación argumentando que los entornos de aprendizajes virtuales constituyen una forma totalmente nueva. Es ahí, donde la Universidad Nacional Experimental de los Llanos Occidentales Ezequiel Zamora (UNELLEZ), visualiza un abanico en oportunidades que ofrece la virtualidad mediante la asimilación e integración de herramientas digitales en las nuevas carreras.

Respaldando lo anterior, las autoras Flores y Garrido (2019a): expresan que "[...] los docentes universitarios tengan iniciativas en el uso de las TIC para gestionar los procesos académicos para aprender a aprender y aprender a enseñar [...]" (pág. 54). Es decir, la necesidad de desarrollar un marco de iniciativas que guíen a los docentes a definir, planificar, organizar, ejecutar estrategias para la enseñanza-aprendizaje en línea, el cual dará cambios significativos en los estudiantes de Licenciatura en Turismo Agroecológico. Los docentes universitarios tenemos que tejer las redes de las iniciativas tecnológicas para aprender y aprender a enseñar así, ayudar en el avance de la educación.

Dando pie a lo anterior, ofrecer a los estudiantes nuevas formas de enseñanza-aprendizaje virtuales, que aporten elementos para la construcción de saberes presenciales. Desde esta configuración, los entornos virtuales, espacios generadores de condiciones propias para construir el conocimiento.

Este tipo de modalidad se caracteriza por la no presencia física de quien enseña y quien aprende, no obstante, se requiere la presencia de un docente que funja en un rol de tutor brinde orientaciones vitales para el estudiante con habilidades en un futuro ser un profesional sistémico con valores, actitudes, aptitudes, es fundamental.

En el Programa de Formación de Grado Licenciatura en Turismo Agroecológico (PFGLTA) se impulsa una nueva mentalidad, utilizando 


\section{Ensayo Original / Original Essay}

herramientas digitales, metodologías, más allá de las tecnologías, la variable de la presencialidad está presente valga la redundancia, mediante las vivencias de los actores sociales conllevando inexorablemente a experiencias innovadoras. Están claros los cuestionamientos: ¿los docentes estamos preparados para un cambio significativo en los procesos enseñanzaaprendizaje virtual?; ¿Existen la formación y capacitación en el área digital y tecnológica del docente?; ¿Es esencial la presencia tecnológica en los estudios académicos universitarios?; ¿Integración entre lo presencialidad y la virtualidad del estudiante-docente?.

En este sentido, el presente ensayo plasmará a lo largo, reflexiones sobre el cambio significativo en los procesos enseñanza-aprendizaje virtual en PFGLTA. La vinculación entre lo presencialidad y la virtualidad estará marcada por las acciones ejercidas por los estudiantes en sus comunidades después de lo aprendido a través del docente en lo virtual.

\section{Desarrollo}

Es indiscutible, nuevas carreras difieren de las tradicionales, haciéndose necesario la integración de una forma articulada de la calidad del proceso enseñanza-aprendizaje virtual debe estar presente en toda su trayectoria. La necesidad de un replanteamiento en cuanto a las aulas, los docentes tenemos que navegar hacia los entornos virtuales, lugares que aportan una mejora educativa facilitando un cambio pedagógico. En este instante, el discípulo asume su protagonismo siendo responsable de su formación en este momento, entra en el escenario la preparación docente mediante el uso de herramientas sincrónicas y asincrónicas para el favorecimiento del proceso integración aprendizaje activo y colaborativo creando redes de conocimientos y aprendizaje.

Dentro este contexto, la Universidad Nacional Experimental de los Llanos Occidentales "Ezequiel Zamora" (UNELLEZ), Barinas, Venezuela, no 
pierde la perspectiva innovadora, postmoderna, crítica, humanística, pertinente ambientalista mediante la enseñanza y el aprendizaje siendo un compromiso de la universidad. Bajo esta perspectiva, la creación de la carrera de Turismo Agroecológico promueve acciones pedagógicas con herramientas tecnológicas donde el docente se apoya para la creación de un ambiente dinámico, participativo y colaborativo donde se aprende y desaprende a través de un entramado de los entornos físicos y virtuales, afianzando el intercambio de saberes, una sinergia entre el docente-estudiante.

\subsection{En reconstrucción de lo presencialidad y virtualidad}

Habiendo transcurrido una década del siglo XXI, la educación en la actualidad debe ser innovada, por lo tanto, la UNELLEZ ofrece la Licenciatura en Turismo Agroecológico hacia una nueva concepción a nivel universitario. El procedimiento enseñanza-aprendizaje virtual con una pincelada de presencialidad en la naciente carrera bajo una nueva naturaleza del conocimiento implica un cambio fundamental pero complejo, es decir, comprender en profundidad la relación gestión sobre percibir, seleccionar, interactuar con la realidad con el conocimiento.

Dada la importancia actual que tiene el Turismo para la preservación del ambiente, el resalte de los encantos turísticos de la región, la UNELLEZ conlleva a la formación de Licenciados en Turismo Agroecológico convirtiéndola en una oferta, de enseñanza-aprendizaje permitiendo modelos, enfoques y teorías desde lo virtual al ambiente. Para el Consejo Directivo de la Universidad Nacional Experimental de los Llanos Occidentales "Ezequiel Zamora" (UNELLEZ, 2017): "[...] es un método de aprendizaje de raíz constructivista, donde se integra el a prender y el enseñar, siendo el inicio del ciclo del acto de aprendizaje la práctica, la vivencia, el descubrimiento, se pone en evidencia la relación formación-implicación-trabajo" (pág. 22). Produciéndose un contexto educativo en el PFGLTA, visualizando un entorno 


\section{Ensayo Original / Original Essay}

sociocultural, socio productivo y socio ambiental, apoyado con un parámetro virtual para que los estudiantes puedan exportar las bellezas naturales mediante herramientas virtuales.

Hoy, segunda década del siglo XXI, la incorporación de tecnologías ha facilitado los procesos de enseñanza-aprendizaje virtual en la adquisición, obtención de información convirtiéndose en una necesidad muy arraigada en la vida diaria, considerándolas como la integración con el entorno. Frente a esto, Castells (2002): dice que "[...] nos estamos refiriendo a un nuevo paradigma tecnológico que tiene dos expresiones fundamentales: una es internet y la otra la capacidad de recodificar los códigos de la materia viva" (pág. 1). Es imprescindible, identificar todas las vertientes digitales, intercambiando información, conocimientos y estableciendo relaciones mediante la interactuación.

Por tal razón, las estrategias para enseñanza-aprendizaje virtual son vitales como lo sustenta Esteban (2009): "las estrategias se suelen clasificar, generalmente, en función de las actividades cognitivas" (pág. 2); haciendo uso de capacidades ofreciendo medios para alcanzar habilidades, conocimientos, destrezas. Es conveniente, un proceso de enseñanza-aprendizaje virtual que permita el planteamiento desde lo virtual al ambiente para la Licenciatura en Turismo Agroecológico.

El uso de los medios tecnológicos donde la educación es personalizada: Un Aprender haciendo, a conocer, a hacer, a convivir y a ser, constituyendo una herramienta esencial de reflexión. En la actualidad, las nuevas tecnologías poseen recursos que al ser utilizados nos facilita la vivencia del entorno virtual agroecológico, natural, cultural, turístico, siempre bajo el enfoque la comprensión de las nuevas herramientas digitales para conquistar el interés en el campo del turismo agroecológico a través de la web.

En tal sentido, el docente debe ser un ciudadano digital para la promocionar sus productos y servicios educativos con el fin de un desempeño 


\section{Ensayo Original / Original Essay}

exitoso de nuestros egresados en Turismo Agroecológico. A través del conocimiento virtual se ha creado espacios geográficos, rutas y circuitos turísticos, específicamente dentro de nuestra universidad, comunidades.

\subsection{Tejiendo las redes}

Los docentes debemos prepáranos para un cambio significativo en los procesos enseñanza-aprendizaje virtual, a este respecto resulta oportuno como docentes las modalidades presenciales como virtuales permiten trabajar y entramar las actividades con las competencias tecnológicas, generando, compartiendo, produciendo, comunicando, debatiendo, buscando.

Axiomáticamente, es esencial la tecnología en los estudios académicos universitarios tal como lo enuncian Flores y Garrido (2019b): cuando existe "[...] la necesidad emergente de capacitar a los docentes universitarios en el uso de las tecnologías, pero no solamente en la adquisición de competencias básicas, será necesario preparar a los docentes en una verdadera integración de las TIC [...]" (pág. 56); recordando que la tecnología por sí sola, no resuelve nada debe estar integrada con diversos factores porque los entornos virtuales como toda innovación tecnológica siguen evolucionando permitiendo la integración de aplicaciones o herramientas externas, vinculación de las redes sociales.

En este sentido, González, Ojeda y Pinos (2020a): mencionan que "esto implica un cambio en los hábitos y materiales educativos, comprender a los nativos digitales (estudiantes) se torna un verdadero desafío por parte de las migrantes digitales (docentes) [...]" (pág. 325); es imperativo, la adaptación del docente a esta nueva forma de enseñar y aprender.

Resulta evidente que el elemento primordial es la tecnología, pero hay que tener presente que no se puede entender los entornos virtuales sin pedagogía. Es imprescindible, identificar otras vertientes que involucran en el proceso del mejoramiento de habilidades y adaptarse a situaciones 


\section{Ensayo Original / Original Essay}

cambiantes.

La incorporación de Tecnología del Aprendizaje y el Contenido (TAC), Tecnología del Empoderamiento y la Participación (TEP), un aglomerado tecnológico digno para comprender las diversas tecnologías que marcan las pautas para ejercer la docencia en Turismo Agroecológico. Como lo surgieren Granados, López, Avello, Luna, Luna y Luna (2014): “la penetración de las TIC - TAC y TEP promete acortar caminos, por su potencial para tender puentes y revertir desigualdades en múltiples ámbitos" (pág. 293). Por ello, es necesario disminuir discrepancias debido al aluvión tecnológico, enfocándonos en la parte docente del ámbito universitario es imprescindible, herramientas donde los jóvenes de empoderan de los conocimientos para la construcción, igualmente los docentes se apropian de las herramientas para conceptuar la enseñanza-aprendizaje virtual.

En este sentido, González, Ojeda y Pinos (2020b): "[...] las actividades académicas ya no son rígidas y es necesario que el docente proponga nuevos métodos e instrumentos innovadores que despierten el aprendizaje del estudiante para la vida" (pág. 325). Esta nueva terminología usada para la educación fomentaría la participación de los jóvenes estudiantes de Turismo Agroecológico, compartiendo ideas, intereses ya sean en lo social, político para la generación de un empoderamiento y, por ende, la concientización de la posición que se ocupa en la sociedad.

La universidad debe ofrecer activos elementos digitales, para la formación integrar de profesionales capaces de desarrollar destrezas/habilidades. Como lo sugiere la Organización de las Naciones Unidas para la Educación, la Ciencia y la Cultura (UNESCO, 2004): "la importancia del contexto, la cultura, la visión y liderazgo, el aprendizaje permanente y los procesos de cambio al momento de planificar la integración de las tecnologías a la capacitación docente" (pág. 14).

La universidad suministra herramientas para ser utilizadas en 


\section{Ensayo Original / Original Essay}

enseñanza-aprendizaje virtual, motivando al docente a que despierte y motive el proceso de la creatividad en los estudiantes para que aprendan haciendo en la práctica, invirtiendo el tiempo en investigan logrando la generación y el compartimiento del aprendizaje colaborativo suscitando experiencias inolvidables en ellos mismos. Procesos de cambio fundamentados en la existencia de una interacción orientada a guiarlos mediante la compresión para que hagan investigación porque el docente es la guía del proceso de aprendizaje.

Indiscutiblemente, formación y capacitación del área digital y tecnológica del docente es un compromiso personal y profesional porque es un aprendiz activo en la construcción de un nuevo ser que espera su guía para darle sentido a la información suministrada. Queda demostrado para Flores y Garrido (2019c): que es necesario "[...] aprender el uso pedagógico de la tecnología que sirva como mediador de nuevos aprendizajes" (pág. 56); debemos surfear las debilidades capaces de ayudar a los jóvenes a desarrollar sus capacidades con las herramientas digitales y que sean idóneos de crear innovaciones presenciales mediante la enseñanza-aprendizaje virtual ofreciéndoles respuestas a qué aprender, cómo aprender.

La integración entre lo presencialidad y la virtualidad del estudiantedocente de la carrera en Turismo Agroecológico, se evidencia de lo que se enseña mediante la metodología y estrategias con recursos virtuales para enseñar al educando a ser creativo y llevar a la practica en su entorno, proyectándose significativamente y adecuado a la realidad. La creación de las diversas rutas agroecológicas por los estudiantes de Turismo Agroecológico es una muestra de la integración entre lo presencialidad y la virtualidad.

\section{Conclusiones}

Es elocuente destacar, requieren una manera apremiante, los entornos virtuales, el compromiso del docente, poniendo en práctica nuevas formas de 


\section{Ensayo Original / Original Essay}

enseñanza-aprendizaje en línea conjugado con lo presencial donde los profesores aprendan habilidades requeridas y así lograr la trasmisión de conocimientos hacia los estudiantes de Licenciatura en Turismo Agroecológico.

La práctica de la construcción del enseñanza-aprendizaje virtualpresencial es un hecho mediante la creación de las rutas turísticas, concebido a través presencial con proyección virtual reflejo de la capacidad de aprender, trabajo en equipo, manejo y de familiarización, diseño e implantación con unidades de producción y desarrollo sustentable mediante la valoración del ambiente.

La incorporación de la tecnología ofrece un abanico de posibilidades que no se puede ignorar y mucho menos privar a las nuevas carreras los conocimientos digitales del cosmos tecnológico abriendo puertas inimaginables.

Es necesario, aprender a usar las herramientas tecnológicaspedagógicas en la educación universitaria del siglo XXI. Finalmente, la formación del uso digital debe ser fomentada por las universidades ofreciendo conectividad para garantizar el conocimiento, manejo y uso de las tecnologías en la vida universitaria en los tiempos actuales.

Las Tecnologías de Información y Comunicación (TIC), la Tecnología del Aprendizaje y el Contenido (TAC), y la Tecnología del Empoderamiento y la Participación (TEP), brindan un sinfín de oportunidades que no se puede ignorar y mucho menos privar a los docentes de los conocimientos del cosmos tecnológico. Los docentes en la aplicación de la enseñanza-aprendizaje virtual deben tener presente la conectividad para adquirir competencia digital.

Hablar de interconexión en la enseñanza-aprendizaje virtual es, presencia de docentes, estudiantes y universidad, pilares fundamentales para su desarrollo. La tecnología en sí, nada hacer, es necesario, el acceso al conocimiento, la información. Por lo anterior, dichos elementos cada vez son 
más importantes en la vida universitaria actual. La virtualidad debe ser fomentada por las universidades ofreciendo conectividad para garantizar el conocimiento, manejo y uso de las tecnologías. La necesidad de un docente conocedor, hábil que debe estar a la vanguardia. Es un cambio tradicional a un docente con protagonismo tecnológico.

En este segundo decenio del presente siglo, la existencia del planteamiento de la modificación del proceso enseñanza-aprendizaje es latente, cambios de pensamiento en la educación universitaria. La integración entre lo presencialidad y la virtualidad, es reto para el docente dar una orientación en la búsqueda académica, enseñar a compartir información teniendo la presencia de la controversia mediante con basamento teórico dando origen a la criticidad. Haciendo entramados para el desarrollo, construcción y conectividad del conocimiento en la formación de competencias en los estudiantes, teniendo la capacidad de desempolvar contenidos académicos del siglo XX para actualizarlos en respuesta a las exigencias actuales.

Y hoy en día, se está comenzando a escribir en una página en blanco, el cambio y el impacto del docente hacia el manejo de herramientas digitales/tecnológicas ocasionado por la pandemia. Esto es solo el comienzo de una ruta de la carrera todavía falta ver los frutos, nuestros egresados, innovadores de un pensamiento y un proceder, distintos del siglo XX, es decir, un antes y un después.

\section{Referencias}

Castells, M. (2002). La dimensión cultural de Internet. Sección 1: Cultura y Sociedad de Conocimiento: Presente y Perspectivas del Futuro. Barcelona, España: FUOC e ICUB.

Esteban, M. (2009). Las estrategias de aprendizaje en el entorno de la Educación a Distancia (EaD). Consideraciones para la reflexión y 
Ensayo Original / Original Essay

el debate. Introducción al estudio de las estrategias y estilos de aprendizaje. RED: Revistas de Educación a Distancia, (7), 1-4, e-ISSN: 1578-7680. Recuperado de:

https://revistas.um.es/red/article/view/25371

Flores, D., \& Garrido, J. (2019a,b,c). Competencias digitales para los nuevos escenarios de aprendizaje en el contexto universitario. Revista Scientific, 4(14), 44-61, e-ISSN: 2542-2987. Recuperado de: https://doi.org/10.29394/Scientific.issn.2542-2987.2019.4.14.2.44-61

González, M., Ojeda, M., \& Pinos, P. (2020a,b). Desafío del Siglo XXI en la educación: dando saltos del TIC-TAC al TEP. Revista Scientific, 5(18), 323-344, e-ISSN: 2542-2987. Recuperado de:

https://doi.org/10.29394/Scientific.issn.2542-2987.2020.5.18.17.323$\underline{344}$

Granados, J., López, R., Avello, R., Luna, D., Luna, E., \& Luna, W. (2014). Las tecnologías de la información y las comunicaciones, las del aprendizaje y del conocimiento y las tecnologías para el empoderamiento y la participación como instrumentos de apoyo al docente de la universidad del siglo XXI. MediSur, 12(1), 289-294, eISSN: 1727-897X. Recuperado de:

http://www.medisur.sld.cu/index.php/medisur/article/view/2751

UNELLEZ (2017). Diseño Curricular: Programa de Formación de Grado. Licenciatura en Turismo Agroecológico (PFGLTA). Barinas, Venezuela: Secretaria Ejecutiva de Desarrollo e Innovación CurricularSEDIC; Universidad Nacional Experimental de los Llanos Occidentales "Ezequiel Zamora".

UNESCO (2004). Las Tecnologías de la información y la comunicación en la formación docente: guía de planificación. Código del documento: ED/HED/TED/3. París, Francia: División de Educación Superior de la Organización de las Naciones Unidas para la Educación, la Ciencia y la 
Instituto Internacional de Investigación y Desarrollo Tecnológico Educativo INDTEC, C.A.

DOI: https://doi.org/10.29394/Scientific.issn.2542-2987.2021.6.19.18.349-365

OAI-PMH: http://www.indteca.com/ojs/index.php/Revista Scientific/oai

Ensayo Original / Original Essay

Cultura.

UNESCO (1998). Declaración Mundial sobre la Educación Superior en el siglo XXI: visión y acción. Revista Educación Superior y Sociedad (ESS), 9(2), 97-113, e-ISSN: 2610-7759. Recuperado de:

https://www.iesalc.unesco.org/ess/index.php/ess3/article/view/171 


\section{Iraima Sofía Gutiérrez Mendoza \\ e-mail: gunenaeli@gmail.com}

Nacida en Libertad, Barinas, estado Barinas, Venezuela,

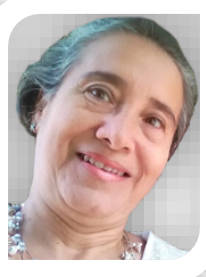
el 11 de diciembre del año 1966. Realicé estudios en la Universidad Nacional Experimental del Táchira (UNET), como Ingeniero Industrial; posteriormente la Maestría en Gerencia Empresas Agrícolas y el Doctorado en Gerencia por la Universidad Yacambú (UNY); Docente en Licenciatura en Turismo Agroecológico; Medicina Veterinaria; Ingeniería Agroindustrial; y Licenciatura en Administración Agropecuaria por la Universidad Nacional Experimental de los Llanos Occidentales Ezequiel Zamora (UNELLEZ), Barinas, Venezuela; Miembro de la Comisión de mejoramiento profesional del Centro de Ingeniero, Táchira. 


\section{Ensayo Original / Original Essay}

\section{Yamirle Mercedes Pérez Girón \\ e-mail: ympg1234@gmail.com}

Nacida en Barinas, estado Barinas, Venezuela, el 20 de

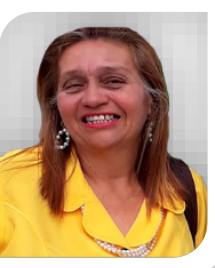
diciembre del año 1964. Realicé estudios en Universidad Nacional Experimental de los Llanos Occidentales Ezequiel Zamora (UNELLEZ), egresada como Licenciado en Administración; también soy Especialista en Planificación; y Magister Scientiarum en Administración; me he desempeñado con el cargo de Auxiliar de Auditoría del Instituto Nacional de la Vivienda (INAVI); Coordinadora del programa sociocultural de Apure para desarrollos sociales en el Ministerio de la Familia; he sido Vicepresidenta de la Junta Directiva del Colegio de Administradores del Estado Apure; Docente fijo (instructor) en la UNELLEZ-VPDS; Programas de Ciencias Sociales y económicas y Ciencias del Agro y del Mar; Tutor Académico y ponente en diversos eventos. 


\section{Ensayo Original / Original Essay}

\section{Antonio José Gutiérrez Mendoza}

e-mail: gutierrezm65@gmail.com

Nacido en Barinas, estado Barinas, Venezuela, el 11 de

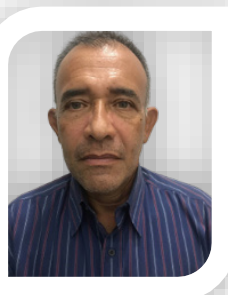
junio del año 1965. Realicé mis estudios de tercer nivel en la Universidad Nacional Experimental de los Llanos Occidentales Ezequiel Zamora (UNELLEZ); titulándome de Ingeniero Agroindustrial; He ejercido trabajos en el Instituto Agrario Nacional (IAN); Actualmente trabajo en Instituto Nacional de Capacitación y Educación Socialista (INCES), como Jefe de Informática; Imparto la docencia en la asignatura de informática en la Misión Sucre y en la UNELLEZ, San Carlos.; siendo Tutor Académico en trabajos de grado, de pregrado de Ingeniería Agroindustrial en Barinas; y profesor-asesor en la Misión Sucre; además de ser ponente en diversos congresos. 